Open Access

\title{
Sepsis prediction in critically ill patients by platelet activation markers on ICU admission: a prospective pilot study
}

Nathalie Layios ${ }^{1,2+}$, Céline Delierneux ${ }^{2 \dagger}$, Alexandre Hego ${ }^{2}$, Justine Huart ${ }^{2}$, Christian Gosset $^{3}$, Christelle Lecut ${ }^{3}$, Nathalie Maes ${ }^{4}$, Pierre Geurts ${ }^{5}$, Arnaud Joly ${ }^{5}$, Patrizio Lancellotti ${ }^{2,6}$, Adelin Albert ${ }^{4}$, Pierre Damas ${ }^{1}$,

André Gothot ${ }^{3}$ and Cécile Oury ${ }^{2^{*}}$

* Correspondence:

cecile.oury@ulg.ac.be

${ }^{\dagger}$ Equal contributors

${ }^{2}$ Laboratory of Thrombosis and Hemostasis, GIGA-Cardiovascular Sciences, University of Liège, Department of Cardiology, University Hospital of Liège, Liège, Belgium

Full list of author information is available at the end of the article

\section{Springer Open}

\begin{abstract}
Background: Platelets have been involved in both immune surveillance and host defense against severe infection. To date, whether platelet phenotype or other hemostasis components could be associated with predisposition to sepsis in critical illness remains unknown. The aim of this work was to identify platelet markers that could predict sepsis occurrence in critically ill injured patients.
\end{abstract}

Methods: This single-center, prospective, observational, 7-month study was based on a cohort of 99 non-infected adult patients admitted to ICUs for elective cardiac surgery, trauma, acute brain injury, and post-operative prolonged ventilation and followed up during ICU stay. Clinical characteristics and severity score (SOFA) were recorded on admission. Platelet activation markers, including fibrinogen binding to platelets, platelet membrane P-selectin expression, plasma soluble CD40L, and platelet-leukocytes aggregates were assayed by flow cytometry at admission and $48 \mathrm{~h}$ later, and then at the time of sepsis diagnosis (Sepsis-3 criteria) and 7 days later for sepsis patients. Hospitalization data and outcomes were also recorded.

Methods: Of the 99 patients, 19 developed sepsis after a median time of 5 days. These patients had a higher SOFA score at admission; levels of fibrinogen binding to platelets (platelet-Fg) and of D-dimers were also significantly increased compared to the other patients. Levels $48 \mathrm{~h}$ after ICU admission no longer differed between the two patient groups. Platelet-Fg \% was an independent predictor of sepsis $(P=0$. 0031). By ROC curve analysis, cutoff point for Platelet-Fg (AUC $=0.75$ ) was $50 \%$. In patients with a SOFA cutoff of 8 , the risk of sepsis reached $87 \%$ when Platelet-Fg levels were above $50 \%$. Patients with sepsis had longer ICU and hospital stays and higher death rate.

Conclusions: Platelet-bound fibrinogen levels assayed by flow cytometry within $24 \mathrm{~h}$ of ICU admission help identifying critically ill patients at risk of developing sepsis.

Keywords: Sepsis, Prediction, Flow cytometry, Platelet markers, Fibrinogen, SOFA, Biomarker

\section{Background}

Despite sustained research on the immune pathophysiology of sepsis, sepsis occurrence remains the leading cause of mortality $(20-50 \%)$ in the intensive care unit (ICU) [1, 2].

(c) The Author(s). 2017 Open Access This article is distributed under the terms of the Creative Commons Attribution 4.0 International License (http://creativecommons.org/licenses/by/4.0/), which permits unrestricted use, distribution, and reproduction in any medium, provided you give appropriate credit to the original author(s) and the source, provide a link to the Creative Commons license, and indicate if changes were made. 
Therefore, the identification of predictive biomarkers of sepsis is instrumental to improve ICU patients' outcome. The Third International Consensus Task Force (Sepsis-3) defines sepsis as a "life-threatening organ dysfunction caused by a dysregulated host response to infection". In this concept, growing experimental and preclinical evidence indicates that platelets could play an active role either in immune surveillance or in the response to infection. Indeed, in addition to their role in hemostasis and thrombosis, several studies in animal models suggest a contribution of platelets to infectious diseases due to their ability to influence innate and adaptive immune responses [3]. First, platelets may act as sentinels of the immune system. They indeed express many major receptors of the innate immune system, including most Toll-like receptors. Platelets are able to recognize molecular features of microbes and secrete many immunomodulatory mediators essential for alerting and recruiting cells of the immune system [4-7]. Second, platelets may contain infection both directly and through functional interactions with immune cells [8]. Platelets produce various antimicrobial molecules, including defensins [9], thrombocidins [10], and kinocidins, and they are able to interact with and kill bacteria directly [11]. For instance, it has been shown that activated platelets facilitate the clearance of adherent Streptococci in experimental infective endocarditis [12]; $\beta$-defensins released from platelets activated by the Staphylococcus aureus $\alpha$-toxin impair bacterial growth and induce neutrophil extracellular trap formation [13]. Platelets also help trap blood pathogens on Kupffer cells in hepatic sinusoids, which limits systemic infection [14]. Notably, platelets express CD40L, an essential player in host defense against infection that mediates interactions between platelets, antigenpresenting cells, and lymphocytes [15].

In overwhelming sepsis, platelets contribute to activation of the procoagulant cascade and ensuing complications linked to microvascular thrombosis and subsequent organ dysfunction [16]. It has been demonstrated that critically ill injured adult patients, such as burn, trauma, or cardiac surgery patients, experience susceptibility to sepsis because of innate and adaptive immune reprogramming due to the insult $[17,18]$. However, whether platelets may participate in dysregulated host response to infection leading to sepsis remains unclear. One recent study showed that immature platelet fractions (IPF) could predict sepsis occurrence in critically ill subjects [19]. Further, in severe trauma, platelet activation and leukocyte-platelet aggregate formation have been incriminated in the pathogenesis of tissue lesions leading to organ failure [20]. The present prospective observational study hypothesized that platelet activation markers triggered by common injuries may help predicting occurrence of sepsis in specific ICU patient populations.

\section{Methods}

\section{Study patients}

This was a single-center, prospective, observational, 7-month study based on a cohort of 99 consecutive adult patients, expected to stay for at least $48 \mathrm{~h}$ in tertiary ICU. Inclusion criteria included elective cardiac surgery (coronary artery bypass grafting or valve replacement), trauma, invasive ventilation $>48 \mathrm{~h}$ for reasons other than sepsis, and acute brain injury (including subarachnoid, subdural, intra-parenchymal hemorrhage, and ischemic stroke). Patients were excluded from the study if they 
received oral or parenteral antibiotics other than for prophylaxis and if they were treated with any immunosuppressive agent except substitutive doses of corticosteroids, suffered from chronic hepatitis B or C, HIV, solid organ, or hematologic proliferative disease.

\section{Characteristics at ICU admission}

Upon admission to ICU, the following baseline characteristics were recorded: gender, age, type of admission (surgical or medical), history of diabetes and cardiovascular disease, previous treatment by vasopressor, prophylactic antibiotics, aspirin, and anticoagulants (anti- $\alpha \operatorname{IIb} \beta 3$ ). The sequential organ failure assessment (SOFA) score was computed. Blood samples were collected within $24 \mathrm{~h}$ (T1) for flow cytometry analyses (see the "Flow cytometry" section below). The following laboratory parameters were also assayed: C-reactive protein (CRP, $\mathrm{mg} / \mathrm{ml})$, fibrinogen $(\mathrm{g} / \mathrm{l})$, partial thromboplastin time (PTT, s), prothrombin time index (\%), platelet count $(\mathrm{k} / \mu \mathrm{l})$, D-dimers $(\mu \mathrm{g} / \mathrm{l})$, and WBC count $(\mathrm{K} / \mu \mathrm{l})$. The ISTH scoring system for overt disseminated intravascular coagulation (DIC) was calculated based on Toh et al. [21].

\section{Follow-up and sepsis occurrence}

Patients were sampled again $48 \mathrm{~h}$ (T2) after admission, on the day of diagnosis of sepsis (Tx), and 7 days later. All blood specimens were analyzed by flow cytometry as in T1. A time line diagram is provided as Additional file 1: Figure S1. Criteria for severe sepsis or septic shock are in agreement with the new definitions of sepsis (Sepsis-3) [22]. For each study patient, the following data were also collected: length of ICU and of hospital stay (days), duration of ventilation (days) if required, administration of vasopressor during ICU admission, antibiotic treatment, use of curative antibiotics, red blood cell transfusion, plasma transfusion and platelet transfusion, and hemofiltration or intermittent hemodialysis during or after ICU stay. In case of death, time was also recorded. In case of discharge from the hospital, follow-up was at least 1 year.

\section{Flow cytometry}

Citrated whole blood was collected through an indwelling arterial catheter. Samples were processed within maximum $1 \mathrm{~h}$ following blood drawing. Platelet activation levels were assessed by measuring the expression of P-selectin (PS), a marker of degranulation, and fibrinogen (Fg) binding, as a result of integrin $\alpha \operatorname{IIb} \beta 3$ activation, on cell surface. Specifically, blood samples were fixed and incubated with peridinin-chlorophyll protein-linked (PerCP)-anti-CD61 antibodies (BD Biosciences), fluorescein isothiocyanate-linked (FITC)anti-fibrinogen antibodies (Dako), and phycoerythrin-linked (PE)-anti-CD62P antibodies (BD Biosciences). Levels of platelet activation markers were determined by recording medians of FITC and PE fluorescence intensity (MFI) in platelets (CD61 positive cells) and percentages (\%) of fibrinogen-positive (FITC) or CD62P-positive (PE) platelets on a FACS Verse flow cytometer (BD Biosciences). Data were analyzed using the BD FACSuite software. Platelets-monocytes and platelets-neutrophils aggregates were analyzed in citrated whole blood samples using an antibody panel, including anti-CD45-V500, anti-CD14APC (monocytes), anti-CD15-PE (neutrophils), and anti-CD61-PerCP. Medians of CD61PerCP fluorescence intensity in CD14-positive and CD15-positive cells, and percentages 
of cells double positive for CD61 and CD14, or CD61 and CD15 were recorded as above. In all cases, threshold of positivity was set by use of marker-specific antibodies or their corresponding IgG isotype controls in blood samples that were left unstimulated or activated with a supra-optimal dose of collagen-related peptide. Plasma was prepared from the citrated whole blood samples to quantify plasma levels of TNF $\alpha$, IL10, sCD40L, IL17A, IL6, IL7, and IFN $\gamma$, all expressed in $\mathrm{pg} / \mathrm{ml}$. Cytokine levels were measured using customized multiplex BD ${ }^{\text {mi }}$ Cytometric Bead Array on the FACSVerse System. Analysis was performed with the FCAP Array ${ }^{\text {rm }}$ software.

\section{Statistics}

Results were expressed as mean and standard deviation for quantitative data and as median and interquartile range (IQR) for durations. For categorical findings, frequency tables were used. The predictive value of sepsis was assessed for each baseline variable by logistic regression analysis. Then variables significant at $P<0.10$ were combined in a stepwise logistic regression analysis to identify independent baseline predictors of sepsis. The odds ratio (OR) with $95 \%$ confidence interval $(95 \% \mathrm{CI})$ and ROC curve analysis with area under the curve (AUC) were used to quantify the ability of the selected predictors to discern patients who will later develop sepsis. The Youden method was applied to define an optimal cutoff point for platelet marker predictors and SOFA score. Comparisons of hospital data and outcomes between septic and non-septic patients were done by the Kruskal-Wallis test for continuous variables and the Fisher exact test for categorical variables. Data recorded on the same patients but at different time points were compared by the Wilcoxon signed rank test. Results were considered significant at the $5 \%$ critical level $(P<0.05)$. All statistical calculations were performed with SAS (version 9.4) and $\mathrm{R}$ (version 3.0.3).

\section{Results}

\section{Baseline characteristics of patients}

The baseline ICU admission characteristics of the 99 study patients are displayed in Additional file 2: Table S1. There were 60 men and 39 women aged $64 \pm 15$ years. The type of admission was surgical for 86 patients, and the main reason was predominantly cardiac surgery (68.7\%). Seventeen patients had a history of diabetes and 79 of cardiovascular disease. Ten patients received vasopressor before admission, 67 received prophylactic antibiotics during surgery, 53 were under aspirin, 3 were under $\alpha I I b \beta 3$ antagonist, and 14 patients were taking anticoagulant (only prophylactic doses of low molecular weight heparin). The mean SOFA score was $6.0 \pm 3.3$. Data of routine biological parameters and flow cytometry results upon admission and $48 \mathrm{~h}$ later are displayed in Additional file 1: Table S2. No difference was evidenced between aspirin $(n=53)$ or anticoagulant users $(n=14)$ and non-users in terms of their biological profile (data not shown).

\section{Sepsis occurrence}

Of the 99 study subjects, 19 (19.2\%) developed sepsis after a median time of 5 [IQR 3-7] days and 80 did not. As seen in Table 1, age, gender, type of admission, history of diabetes, use of vasopressor, anti-platelet, or anticoagulation medication use were not associated 
Table 1 Predictive value of patient demographic and baseline clinical data for sepsis development during ICU stay

\begin{tabular}{|c|c|c|c|}
\hline \multirow[t]{2}{*}{ Variable } & \multicolumn{2}{|c|}{ Development of sepsis ${ }^{a}$} & \multirow[t]{2}{*}{$P$ value $^{b}$} \\
\hline & No $(N=80)$ & Yes $(N=19)$ & \\
\hline Age (years) & $65 \pm 15$ & $62 \pm 15$ & 0.46 \\
\hline Gender & & & 0.80 \\
\hline Male & $48(80)$ & $12(20)$ & \\
\hline Female & $32(82.1)$ & $7(17.9)$ & \\
\hline Category of admission & & & 0.70 \\
\hline Surgical & $70(81.4)$ & $16(18.6)$ & \\
\hline Medical & $10(76.9)$ & $3(23.1)$ & \\
\hline Reason for admission & & & 0.0052 \\
\hline Cardiac surgery & $61(89.7)$ & $7(10.3)$ & \\
\hline Acute brain injury & $6(50)$ & $6(50)$ & \\
\hline Trauma & $10(76.9)$ & $3(23.1)$ & \\
\hline Ventilation $>48 \mathrm{~h}$ & $3(50)$ & $3(50)$ & \\
\hline \multicolumn{4}{|l|}{ Score at admission } \\
\hline SOFA & $5.2 \pm 2.7$ & $9.6 \pm 3.1$ & $<0.0001$ \\
\hline Diabetes & & & 0.62 \\
\hline Yes & $13(76.5)$ & $4(23.5)$ & \\
\hline No & $67(81.7)$ & $15(18.3)$ & \\
\hline Cardiovascular disease & & & 0.012 \\
\hline Yes & $68(86.1)$ & $11(13.9)$ & \\
\hline No & $12(60)$ & $8(40)$ & \\
\hline Vasopressor before the admission & & & 0.091 \\
\hline Yes & $6(60)$ & $4(40)$ & \\
\hline No & $74(83.2)$ & $15(16.8)$ & \\
\hline Prophylactic antibiotics & & & 0.0005 \\
\hline Yes & $61(91)$ & $6(9)$ & \\
\hline No & $19(59.4)$ & $13(40.6)$ & \\
\hline Aspirin & & & 0.93 \\
\hline Yes & $43(81.1)$ & $10(18.9)$ & \\
\hline No & $37(80.4)$ & $9(19.6)$ & \\
\hline Anticoagulant & & & 0.24 \\
\hline Yes & $13(92.9)$ & $1(7.1)$ & \\
\hline No & 67 (78.8) & $18(21.1)$ & \\
\hline
\end{tabular}

${ }^{a}$ Means \pm SD for quantitative variable and numbers (\%) for qualitative parameters

bogistic regression

with sepsis development. By contrast, patients who later developed sepsis presented with higher SOFA score at admission. They were also predominantly admitted for acute brain surgery or prolonged ventilation and lacked prophylactic antibiotics prior to admission. Complementary results of septic compared to non-septic patients are shown in Additional file 2: Table S3.

When considering laboratory tests and flow cytometry parameters recorded within $24 \mathrm{~h}$ of admission to ICU (Table 2), D-dimers and fibrinogen binding to platelets (platelet-Fg expressed as MFI or \%) were markedly higher $(P<0.001)$ in patients who later 
Table 2 Predictive value of laboratory tests assessed at admission for sepsis development during

\begin{tabular}{|c|c|c|c|}
\hline \multirow[t]{2}{*}{$\begin{array}{l}\text { Variable } \\
\text { nat }\end{array}$} & \multicolumn{2}{|c|}{ Development of sepsis ${ }^{a}$} & \multirow[t]{2}{*}{$P$ value $^{\mathrm{b}}$} \\
\hline & No $(N=80)$ & Yes $(N=19)$ & \\
\hline \multicolumn{4}{|l|}{ Routine } \\
\hline $\mathrm{CRP}(\mathrm{mg} / \mathrm{L})$ & $14.1 \pm 37.7$ & $29.1 \pm 61.7$ & 0.053 \\
\hline Fibrinogen ( $g / L)$ & $2.6 \pm 0.99$ & $3.3 \pm 2.0$ & 0.11 \\
\hline $\mathrm{PTT}(\mathrm{s})$ & $14.4 \pm 1.7$ & $14.4 \pm 3.3$ & 0.78 \\
\hline Prothrombin Time Index (\%) & $66.3 \pm 15.6$ & $69.7 \pm 19.4$ & 0.61 \\
\hline Platelet count $\left(10^{3} / \mu \mathrm{L}\right)$ & $124 \pm 55$ & $133 \pm 84$ & 0.59 \\
\hline D-dimers $(\mu \mathrm{g} / \mathrm{L})$ & $2617 \pm 6353$ & $4456 \pm 4957$ & 0.0032 \\
\hline ISTH score & $1.6 \pm 1.3$ & $2.6 \pm 0.9$ & 0.041 \\
\hline White blood cell count $\left(10^{3} / \mu \mathrm{L}\right)$ & $10.0 \pm 4.4$ & $11.1 \pm 5.2$ & 0.42 \\
\hline \multicolumn{4}{|l|}{ Flow cytometry } \\
\hline TNF-a (pg/mL) & $0.17 \pm 0.8$ & $0.65 \pm 1.5$ & 0.091 \\
\hline IL-10 (pg/mL) & $19.4 \pm 90$ & $9.4 \pm 16.2$ & 0.50 \\
\hline $\mathrm{sCD} 40 \mathrm{~L}(\mathrm{pg} / \mathrm{mL})$ & $88.8 \pm 81.8$ & $53.3 \pm 43.6$ & 0.89 \\
\hline IL-17A (pg/mL) & $9.1 \pm 12.2$ & $7.6 \pm 15.5$ & 0.31 \\
\hline IL-6 (pg/mL) & $459 \pm 2673$ & $162 \pm 164$ & 0.94 \\
\hline IL-7 (pg/mL) & $2.9 \pm 3.7$ & $1.4 \pm 1.7$ & 0.65 \\
\hline $\mathrm{IFN}-\gamma(\mathrm{pg} / \mathrm{mL})$ & $0.14 \pm 0.91$ & 0 & 0.99 \\
\hline Platelet-Fg (\%) & $28.1 \pm 27.8$ & $56.5 \pm 31.2$ & 0.0054 \\
\hline Platelet-Fg (MFI) & $1770 \pm 1266$ & $2752 \pm 1359$ & 0.0026 \\
\hline Platelet-PS (\%) & $2.9 \pm 2.4$ & $3.5 \pm 2.7$ & 0.61 \\
\hline Platelet-PS (MFI) & $29.8 \pm 15.1$ & $37 \pm 18.3$ & 0.068 \\
\hline Platelets-neutrophils (\%) & $3.4 \pm 5$ & $4.4 \pm 6.2$ & 0.72 \\
\hline Platelets-neutrophils (CD61 MFI) & $313 \pm 127$ & $323 \pm 137$ & 0.72 \\
\hline Platelets-monocytes (\%) & $19.8 \pm 23.1$ & $22.1 \pm 25.5$ & 0.73 \\
\hline Platelets-monocytes (CD61 MFI) & $1413 \pm 2462$ & $1569 \pm 3182$ & 0.82 \\
\hline
\end{tabular}

Null values for TNF- $a$ and IFN- $\gamma$ correspond to values under the level of detection $(3.8 \mathrm{pg} / \mathrm{ml})$

Platelet-Fg platelet-bound fibrinogen, platelet-PS, platelets expressing P-selectin on their surface, MFI median fluorescence intensity, \% percentage of positive cells for the indicated marker

${ }^{a}$ Results are expressed as means \pm SD

${ }^{b}$ Logistic regression

developed sepsis. To a lesser extent, ISTH DIC score $(P<0.05)$ also differed between septic and non-septic patients. Interestingly, levels of sCD40L, P-selectin on circulating platelets (MFI or \%), platelets-monocytes, and platelets-neutrophils aggregates were not associated with sepsis occurrence. Platelet-Fg correlated weakly with platelet P-selectin $(r=0.32378, P=0.0011, N=98)$, and plasma levels of D-dimers $(r=0.35502, P=0.0004$, $N=96)$ and fibrinogen $(r=0.34592, P=0.0005, N=98)$. No significant correlation was found with platelet count $(r=0.071, P=0.49, N=98)$, sCD40L $(r=-0.10377, P=0.3222$, $N=93$ ), or cytokine levels. Flow cytometry parameters recorded $48 \mathrm{~h}$ after admission were not associated with sepsis occurrence, although a tendency $(P<0.10)$ remained for platelet-Fg (data not shown). When looking at serial platelet-Fg levels in patients who developed sepsis, a significant increase was observed and a peak was reached on the day of sepsis (Fig. 1). By contrast, sCD40L remained fairly stable as sepsis developed (Additional file 1: Figure S2). D-dimers and platelet P-selectin levels 


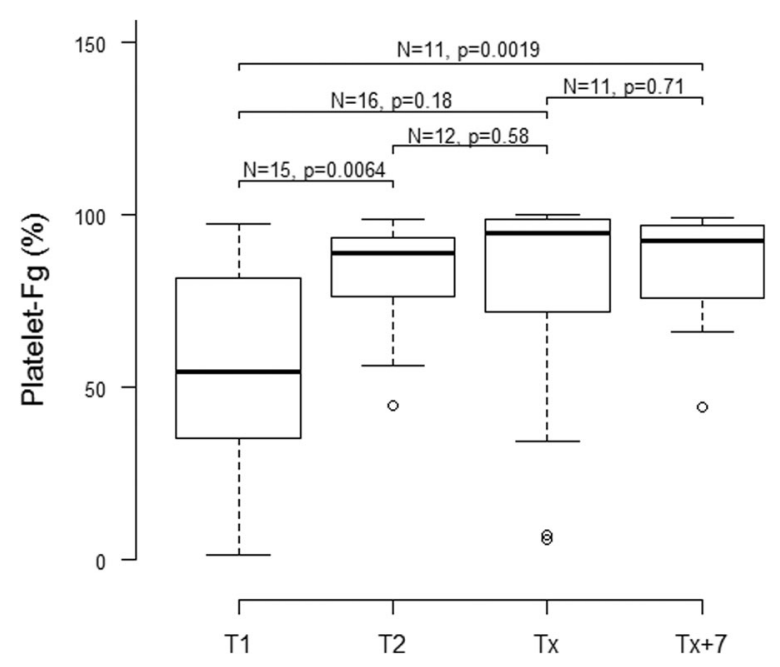

Fig. 1 Serial measurements of levels of platelet-bound fibrinogen (platelet-Fg) for patients who developed sepsis. Platelet-Fg levels were analyzed by flow cytometry on the day of ICU admission (T1), after $48 \mathrm{~h}(T 2)$, at the time of sepsis diagnosis $(T x)$, and 7 days later $(T x+7)$. Median values of percentages of Fg-positive platelets and IQR are shown ( $P$ value: Kruskal-Wallis test)

increased significantly from $\mathrm{T} 2$ to the time of sepsis diagnosis (Additional file 1: Figure S2).

Platelet markers at admission and sepsis prediction

All potential predictors of sepsis $(P<0.10)$ recorded at ICU admission $(\mathrm{T} 1)$ were combined into a stepwise logistic regression analysis. As diagnosis of sepsis includes organ dysfunction, SOFA score was not included in our regression model. It turned out that platelet-Fg \% levels at T1 $(P=0.0031)$ and admission for acute brain injury $(P=0.012)$ were the only independent predictors of sepsis occurrence. By ROC curve analysis (Fig. 2), an optimal cutoff point equal to 50\% was derived for platelet-Fg \% $(\mathrm{AUC}=0.75)$ to discern patients who will later develop sepsis from those who will not. The number of patients who developed sepsis was respectively equal to $13(46.4 \%)$ for the 28 patients with platelet-Fg $>50 \%$ and to $6(8.6 \%)$ for the 70 patients with platelet-Fg $<50 \%$ (data missing for one patient). As shown in Table 3, when accounted for SOFA score at admission (cutoff value of 8), in patients with elevated SOFA and platelet-Fg $>50 \%$, the risk of sepsis rose up to $85.7 \%$. By contrast, in patients with low SOFA and platelet-Fg $<50 \%$, the occurrence of sepsis was almost negligible (3.8\%).

\section{Discussion}

The major findings of this study concern the clear relationship between patient levels of fibrinogen binding to circulating platelets (platelet-Fg) measured upon ICU admission and sepsis occurrence, regardless of the patient's baseline clinical characteristics. In particular, the study demonstrated that for patients presenting a SOFA score $\geq 8$, platelet-Fg $\%$ level above 50 predicted sepsis with a high accuracy. Importantly, neither platelet membrane-bound P-selectin expression plasma levels of sCD40L nor any other standard hemostasis parameter showed similar 


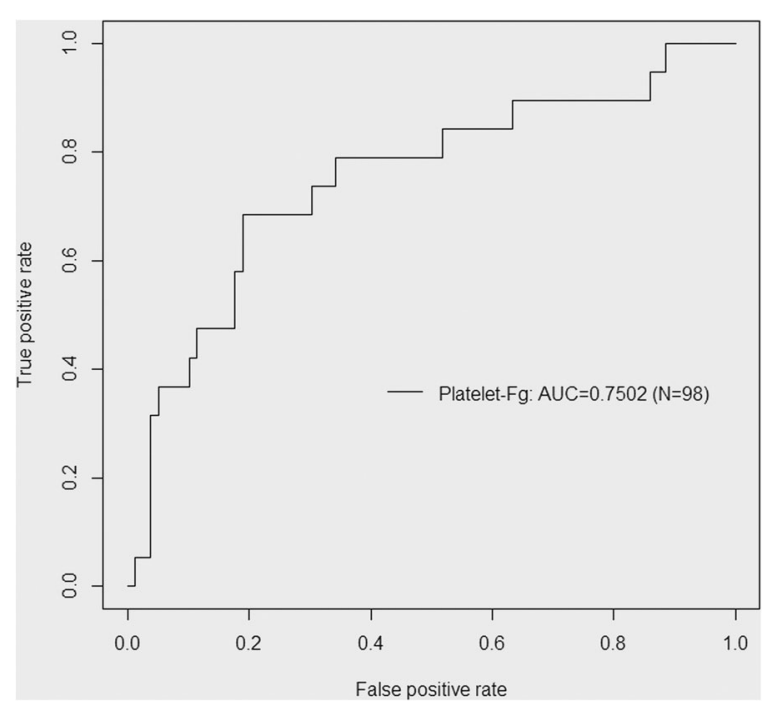

Fig. 2 Predictive value of platelet-Fg (\%) obtained at admission. ROC curve analysis of sepsis prediction based on platelet-Fg is shown

predictive value as platelet-Fg. The optimal timing of measurement was also determined since only levels obtained within $24 \mathrm{~h}$ after ICU admission and not $48 \mathrm{~h}$ later were associated with sepsis occurrence, thus saving blood sampling in future studies. Platelet-Fg levels can be obtained in $1 \mathrm{~h}$ by using whole blood flow cytometry in unstimulated samples. Thus, this work provides the clinician with a simple and practical tool to assess the risk of sepsis in critically ill patients admitted to the ICU.

To date, several clinical studies investigated platelet markers in various conditions of critical illness. However, none of them searched for a potential association of these platelet markers with a risk for sepsis. Most of these studies described altered platelet phenotype in injured patients, characterized by either differential expression of platelet activation markers or platelet dysfunction as compared to healthy controls [23-28]. In ischemic stroke, two studies showed increased expression of platelet Pselectin and fibrinogen binding to platelets as compared to controls [25, 29]. The latter finding is interesting in view of our results, in particular since predisposition to severe pneumonia is clinically well established in such patients [30, 31]. Unfortunately, no association was searched between high levels of the biomarker and pneumonia. Several other clinical studies focusing on platelets as potential biomarkers for

Table 3 Risk stratification of patients according to sepsis development during ICU stay

\begin{tabular}{|c|c|c|c|c|}
\hline \multirow{3}{*}{ Development of sepsis } & \multicolumn{2}{|c|}{ SOFA $<8$} & \multicolumn{2}{|c|}{$\underline{\text { SOFA } \geq 8}$} \\
\hline & \multicolumn{2}{|c|}{ Platelet-Fg (\%) } & \multicolumn{2}{|c|}{ Platelet-Fg (\%) } \\
\hline & $<50$ & $\geq 50$ & $<50$ & $\geq 50$ \\
\hline Yes $(N=19)$ & 2 & 1 & 4 & 12 \\
\hline No $(N=79)$ & 51 & 13 & 13 & 2 \\
\hline Total & 53 & 14 & 17 & 14 \\
\hline Risk of sepsis (\%) & 3.8 & 7.1 & 23.5 & 85.7 \\
\hline
\end{tabular}

SOFA score and Platelet-Fg (\%) plasma levels recorded on admission 
sepsis diagnosis and prognostication have been carried out but almost all concerned patients with sepsis as an inclusion criterion [32, 33].

Despite multiple experimental data demonstrating antimicrobial activity of platelets and a role for platelet aggregation in limiting pathogen growth and dissemination in the vasculature $[3,6]$, direct clinical evidence from human studies was lacking and there are no epidemiologic data showing that platelet function inhibition affects sepsis prediction or prognosis. The present observational prospective study provides the first clinical evidence that, in patients with critical illness and related organ dysfunction, platelets may intervene in the dysregulated host response to infection leading to sepsis. Although demonstration of a causal link requires further investigation, we speculate that injury-associated platelet activation and subsequent fibrinogen binding may alter platelet ability to recognize bacterial components, some of which are ligands of $\alpha \operatorname{IIb} \beta 3$ [34, 35], and affect their ability to alert and recruit cells of the immune system [8]. Our observation that plateletFg weakly correlates with D-dimer levels suggests that fibrinogen binding to platelets and the activation of coagulation could be driven by the same factors. In injured patients, plasma fibrinogen would both bind platelets and be actively converted into fibrin; fibrinolysis would then increase D-dimer levels.

Antiplatelet drugs have beneficial and detrimental effects in systemic inflammation and in organ dysfunction, as shown in preclinical models and in humans [36-38]. Their usage has been variably associated with sepsis prognosis [39, 40]. In this study, we found no protective effect of aspirin against sepsis [41]. Our results are in line with a recent propensity-based analysis of 972 patients admitted for sepsis in which no association between aspirin therapy and sepsis prognosis could be evidenced [42]. Our results however differ in that they encompassed the period before sepsis, a period during which the abovementioned authors could not assess the potential benefits of aspirin. In addition, we could not find any association between aspirin therapy and the levels of platelet activation, which suggests that platelet activation pathways independent of thromboxane $\mathrm{A}_{2}$ production could be involved in the patient's platelet response to injury.

\section{Limitations}

The study has a number of limitations. The small sample size and the predominance of elective surgical patients call for caution when interpreting results. Also, possible confounders such as immunomodulatory properties of anesthetic drugs were not taken into account at this stage. The findings of this pilot study call for a confirmatory prospective evaluation focusing on fibrinogen levels on platelets in a larger cohort. In our study, the platelet activation markers analyzed, namely levels of fibrinogen, platelet P-selectin expression, platelets-leukocytes aggregates, and SCD40L, behaved differently in their ability to predict sepsis development, which might reflect differences in platelet activation mechanisms or sequences. It has indeed been proposed that platelet activation, in terms of P-selectin expression and fibrinogen binding, and release of immunological molecules (sCD40L, RANTES) result from independent signaling pathways [43]. The utility of other markers, such as platelet microparticles or soluble glycoprotein VI, should be 
analyzed, since the latter is shed from platelet surface and increases in patients with DIC [44].

\section{Conclusions}

In critically ill patients with comorbidities and post-trauma or post-surgical injury, platelet abnormalities are associated with altered host defense mechanisms. We actually found that admission levels of fibrinogen binding to platelets of ICU patients were predictive of later sepsis acquisition. Combining it with stratification based on SOFA score at admission has a higher predictive ability. Hence, our observations could trigger nonspecific preventive interventions such as better supportive care or prophylactic antibiotics as well as research aiming at developing a specific therapeutic tool. Also, the fact that the identified marker was independent of aspirin use might have important future therapeutic implications regarding its actual worldwide implementation of primary or secondary prophylaxis.

\section{Additional files}

Additional file 1: Figure S1. Follow-up and sepsis occurrence. Timeline of samplings. Figure S2. Serial measurements of platelet markers and D-dimers for patients who developed sepsis. (DOCX $49 \mathrm{~kb}$ )

Additional file 2: Table S1. Baseline clinical characteristics of study patients ( $n=99)$. Table S2. Baseline and 48-h biological characteristics of study patients $(n=99)$. Table S3. Comparison of ICU- and hospital-related characteristics of patients with and without sepsis. (PDF $157 \mathrm{~kb}$ )

\section{Abbreviations}

AUC: Area under curve; Cl: Confidence interval; CRP: C-reactive protein; Fg: Fibrinogen; MFI: Median of fluorescence intensity; OR: Odds ratio; PS: P-selectin; ROC: Receiver operating characteristic; SOFA: Sequential organ failure assessment

\section{Acknowledgements}

The authors wish to thank the GIGA core facilities (Imaging and flow cytometry) for logistic and technical support.

\section{Funding}

The project was supported by grants from the FNRS (Fonds National pour la Recherche Scientifique FNRS-FRSM 3.4611.11, CDR J.0043.13) and by the French Community of Belgium (FSRC-12/13, ARC-SF 12/14-05). C.L. was a postdoctoral researcher at the FNRS. C.D. was supported by a "Fond pour la recherche industrielle et agricole" fellowship. C.O. is a senior research associate at FNRS. The authors thank BD Biosciences, Europe, for their support in providing the reagents for this study.

\section{Availability of data and materials}

The dataset supporting the conclusions of this article is included within the article and its additional files.

\section{Authors' contributions}

$\mathrm{CD}, \mathrm{AH}, \mathrm{CL}, \mathrm{CG}, \mathrm{AJ}, \mathrm{PG}, \mathrm{JH}, \mathrm{NL}, \mathrm{NM}$, and $\mathrm{CO}$ performed the experiments and analyzed the data. NL, CO, AG, CD, PL, $\mathrm{NM}, \mathrm{AA}$, and PD contributed to the experimental design, analysis, and interpretation of data. NL recruited the patients. $\mathrm{NL}, \mathrm{CO}$, and CD wrote the manuscript. All authors read and approved the final manuscript.

\section{Ethics approval and consent to participate}

The experimental protocol was approved by the ethics committee of the University of Liège Academic Hospital (CHU) (reference number B707201111981). Written informed consent was obtained from each participant or next of kin prior to sampling in agreement with the recommendations of the Declaration of Helsinki for investigations involving human subjects.

\section{Consent for publication \\ Not applicable.}

\section{Competing interests}

The authors declare that they have no competing interests.

\section{Author details}

${ }^{1}$ Department of General Intensive Care, University Hospital of Liège, Liège, Belgium. '2Laboratory of Thrombosis and Hemostasis, GIGA-Cardiovascular Sciences, University of Liège, Department of Cardiology, University Hospital of Liège, Liège, Belgium. ${ }^{3}$ Laboratory of Hematology, University Hospital of Liège, Liège, Belgium. ${ }^{4}$ Department of Biostatistics and Medico-Economic Information, University Hospital of Liège, Liège, Belgium. ${ }^{5}$ Systems and Modeling, Department 
of Electrical Engineering and Computer Science, University of Liège, Liège, Belgium. ${ }^{6}$ Gruppo Villa Maria Care and Research, Anthea Hospital, Bari, Italy.

Received: 13 January 2017 Accepted: 3 July 2017

Published online: 12 July 2017

\section{References}

1. Angus DC, van der Poll T (2013) Severe sepsis and septic shock. N Engl J Med 369:840-851

2. Hotchkiss RS, Monneret G, Payen D (2013) Immunosuppression in sepsis: a novel understanding of the disorder and a new therapeutic approach. Lancet Infect Dis 13:260-268

3. Morrell CN, Aggrey AA, Chapman LM, Modjeski KL (2014) Emerging roles for platelets as immune and inflammatory cells. Blood 123:2759-2767

4. Clark SR, Ma AC, Tavener SA, McDonald B, Goodarzi Z, Kelly MM et al (2007) Platelet tlr4 activates neutrophil extracellular traps to ensnare bacteria in septic blood. Nat Med 13:463-469

5. Aslam R, Speck ER, Kim M, Crow AR, Bang KW, Nestel FP et al (2006) Platelet toll-like receptor expression modulates lipopolysaccharide-induced thrombocytopenia and tumor necrosis factor-alpha production in vivo. Blood 107:637-641

6. Semple JW, Italiano JE Jr, Freedman J (2011) Platelets and the immune continuum. Nat Rev Immunol 11:264-274

7. Weyrich AS, Dixon DA, Pabla R, Elstad MR, Mclntyre TM, Prescott SM et al (1998) Signal-dependent translation of a regulatory protein, bcl-3, in activated human platelets. Proc Natl Acad Sci U S A 95:5556-5561

8. Thomas MR, Storey RF (2015) The role of platelets in inflammation. Thromb Haemost 114:449-458

9. Yeaman MR, Bayer AS, Koo SP, Foss W, Sullam PM (1998) Platelet microbicidal proteins and neutrophil defensin disrupt the staphylococcus aureus cytoplasmic membrane by distinct mechanisms of action. J Clin Invest 101:178-187

10. Yeaman MR, Tang YQ, Shen AJ, Bayer AS, Selsted ME (1997) Purification and in vitro activities of rabbit platelet microbicidal proteins. Infect Immun 65:1023-1031

11. Kerrigan SW, Douglas I, Wray A, Heath J, Byrne MF, Fitzgerald D et al (2002) A role for glycoprotein Ib in Streptococcus sanguis-induced platelet aggregation. Blood 100:509-516

12. Dankert J, van der Werff J, Zaat SA, Joldersma W, Klein D, Hess J (1995) Involvement of bactericidal factors from thrombin-stimulated platelets in clearance of adherent viridans streptococci in experimental infective endocarditis. Infect Immun 63:663-671

13. Kraemer BF, Campbell RA, Schwertz H, Cody MJ, Franks Z, Tolley ND et al (2011) Novel anti-bacterial activities of beta-defensin 1 in human platelets: suppression of pathogen growth and signaling of neutrophil extracellular trap formation. PLoS Pathog 7:e1002355

14. Wong CH, Jenne CN, Petri B, Chrobok NL, Kubes P (2013) Nucleation of platelets with blood-borne pathogens on kupffer cells precedes other innate immunity and contributes to bacterial clearance. Nat Immunol 14:785-792

15. Dewitte A, Tanga A, Villeneuve J, Lepreux S, Ouattara A, Desmouliere A et al (2015) New frontiers for platelet cd154. Exp Hematol Oncol 4:6

16. de Stoppelaar SF, van 't Veer C, van der Poll T (2014) The role of platelets in sepsis. Thromb Haemost 112:666-677

17. Paterson HM, Murphy TJ, Purcell EJ, Shelley O, Kriynovich SJ, Lien E et al (2003) Injury primes the innate immune system for enhanced toll-like receptor reactivity. J Immunol 171:1473-1483

18. Boldt J, Menges T, Wollbruck M, Sonneborn S, Hempelmann G (1994) Platelet function in critically ill patients. Chest 106:899-903

19. De Blasi RA, Cardelli P, Costante A, Sandri M, Mercieri M, Arcioni R (2013) Immature platelet fraction in predicting sepsis in critically ill patients. Intensive Care Med 39:636-643

20. Ogura H, Kawasaki T, Tanaka H, Koh T, Tanaka R, Ozeki Y et al (2001) Activated platelets enhance microparticle formation and platelet-leukocyte interaction in severe trauma and sepsis. J Trauma 50:801-809

21. Toh CH, Hoots WK, ISTH SSCoDICot (2007) The scoring system of the scientific and standardisation committee on disseminated intravascular coagulation of the international society on thrombosis and haemostasis: a 5-year overview. J Thromb Haemost 5:604-606

22. Singer M, Deutschman CS, Seymour CW, Shankar-Hari M, Annane D, Bauer M et al (2016) The third international consensus definitions for sepsis and septic shock (sepsis-3). JAMA 315:801-810

23. Jacoby RC, Owings JT, Holmes J, Battistella FD, Gosselin RC, Paglieroni TG (2001) Platelet activation and function after trauma. J Trauma 51:639-647

24. Davies GR, Mills GM, Lawrence M, Battle C, Morris K, Hawkins K et al (2014) The role of whole blood impedance aggregometry and its utilisation in the diagnosis and prognosis of patients with systemic inflammatory response syndrome and sepsis in acute critical illness. PLoS One 9:e108589

25. Fateh-Moghadam S, Htun P, Tomandl B, Sander D, Stellos K, Geisler T et al (2007) Hyperresponsiveness of platelets in ischemic stroke. Thromb Haemost 97:974-978

26. Gielen CL, Brand A, van Heerde WL, Stijnen T, Klautz RJ, Eikenboom J (2016) Hemostatic alterations during coronary artery bypass grafting. Thromb Res 140:140-146

27. Li N, Astudillo R, Ivert T, Hjemdahl P (2003) Biphasic pro-thrombotic and inflammatory responses after coronary artery bypass surgery. J Thromb Haemost 1:470-476

28. Windelov NA, Ostrowski SR, Johansson PI, Wanscher M, Larsen CF, Sorensen AM et al (2015) Circulating levels of platelet alpha-granule cytokines in trauma patients. Inflamm Res 64:235-241

29. Tsai NW, Chang WN, Shaw CF, Jan CR, Chang HW, Huang CR et al (2009) Levels and value of platelet activation markers in different subtypes of acute non-cardio-embolic ischemic stroke. Thromb Res 124:213-218

30. Hilker R, Poetter C, Findeisen N, Sobesky J, Jacobs A, Neveling M et al (2003) Nosocomial pneumonia after acute stroke: Implications for neurological intensive care medicine. Stroke 34:975-981

31. Meisel C, Schwab JM, Prass K, Meisel A, Dirnagl U (2005) Central nervous system injury-induced immune deficiency syndrome. Nat Rev Neurosci 6:775-786 
32. Russwurm S, Vickers J, Meier-Hellmann A, Spangenberg P, Bredle D, Reinhart K et al (2002) Platelet and leukocyte activation correlate with the severity of septic organ dysfunction. Shock 17:263-268

33. Mosad E, Elsayh Kl, Eltayeb AA (2011) Tissue factor pathway inhibitor and p-selectin as markers of sepsis-induced non-overt disseminated intravascular coagulopathy. Clin Appl Thromb Hemost 17:80-87

34. Cox D, Kerrigan SW, Watson SP (2011) Platelets and the innate immune system: mechanisms of bacterial-induced platelet activation. J Thromb Haemost 9:1097-1107

35. Hamzeh-Cognasse H, Damien P, Chabert A, Pozzetto B, Cognasse F, Garraud O (2015) Platelets and infections-complex interactions with bacteria. Front Immunol 6:82

36. Eisen DP, Reid D, McBryde ES (2012) Acetyl salicylic acid usage and mortality in critically ill patients with the systemic inflammatory response syndrome and sepsis. Crit Care Med 40:1761-1767

37. de Stoppelaar SF, van 't Veer C, van der Poll T (2014) The role of platelets in sepsis. Thromb Haemost. 112.

38. Losche W, Boettel J, Kabisch B, Winning J, Claus RA, Bauer M (2012) Do aspirin and other antiplatelet drugs reduce the mortality in critically ill patients? Thromb 2012:720254

39. Winning J, Neumann J, Kohl M, Claus RA, Reinhart K, Bauer M et al (2010) Antiplatelet drugs and outcome in mixed admissions to an intensive care unit. Crit Care Med 38:32-37

40. Winning J, Reichel J, Eisenhut Y, Hamacher J, Kohl M, Deigner HP et al (2009) Anti-platelet drugs and outcome in severe infection: clinical impact and underlying mechanisms. Platelets 20:50-57

41. Tsai MJ, Ou SM, Shih CJ, Chao PW, Wang LF, Shih YN et al (2015) Association of prior antiplatelet agents with mortality in sepsis patients: a nationwide population-based cohort study. Intensive Care Med 41:806-813

42. Wiewel MA, de Stoppelaar SF, van Vught LA, Frencken JF, Hoogendijk AJ, Klein Klouwenberg PM et al (2016) Chronic antiplatelet therapy is not associated with alterations in the presentation, outcome, or host response biomarkers during sepsis: a propensity-matched analysis. Intensive Care Med 42:352-360

43. Antczak AJ, Singh N, Gay SR, Worth RG (2010) lgg-complex stimulated platelets: a source of scd40l and rantes in initiation of inflammatory cascade. Cell Immunol 263:129-133

44. Al-Tamimi M, Grigoriadis G, Tran H, Paul E, Servadei P, Berndt MC et al (2011) Coagulation-induced shedding of platelet glycoprotein vi mediated by factor xa. Blood 117:3912-3920

\section{Submit your manuscript to a SpringerOpen ${ }^{\circ}$ journal and benefit from:}

- Convenient online submission

- Rigorous peer review

- Open access: articles freely available online

- High visibility within the field

- Retaining the copyright to your article 